\title{
Electron Microscopy and Single Particle Analysis of a Mycobacterial Preprotein Translocation, ATPase
}

\author{
C.G Savva*, A. Arulandu**, J. Sacchettini** and A. Holzenburg*,** \\ *Microscopy \& Imaging Center (MS 2257) and Dept.Biology (MS 3258), Texas A\&M University, \\ College Station, TX 77843 \\ **Dept. Biochem. \& Biophysics (MS 2128), Texas A\&M University, College Station, TX 77843
}

Protein translocation across the inner membrane of bacteria is facilitated by the Sec system which is composed by the cytoplasmic ATPase SecA and the integral membrane proteins SecY, E and G that form the heterotrimeric complex SecYEG [1]. Another chaperone, SecB, delivers preproteins destined to be secreted by the Sec system to SecA [2]. The crystal structure of Bacillus subtillus SecA was recently solved to $3 \AA$ resolution [3]. The proposed functional oligomeric state is a homodimer although evidence has suggested that monomeric [4] and tetrameric [5] states are possible. The 10 A electron crystallographic structure of SecYEG [6] further suggests that SecYEG exists as a dimer whilst others had previously reported a tetrameric state [7]. Thus unless the structure of the entire complex of the translocase machine, SecA-SecYEG, is solved one cannot conclude how the active translocon functions. Combining low resolution EM data with the X-ray data can provide a complete picture of the translocation process. Single particle analysis allows structures to be solved to better than $10 \breve{A}$ without the need for crystallization which can select for oligomeric states that display higher intrinsic symmetries (e.g. tetramers vs. monomers). Here we report the first EM-derived structure of Mycobacterium tuberculosis SecA at $23 \AA$ resolution, which constitutes the first step along the path to establish the translocon structure. The 3D structure indicates that SecA exists as a tetramer in solution with D2 symmetry and measures approximately $140 \AA \times 100 \AA$. The addition of substrate and analogues thereof (ATP, AMP-PNP) results in major conformational changes that cause the protein to aggregate as monitored by EM. Preprotein localization studies using nanogold labeling are currently under way.

Purified SecA was prepared for EM in a high-salt buffer (4M sodium formate). It is interesting to note that only at this high concentration of this specific salt could monodispersed particles be observed with out any aggregates. Specimens were prepared on carbon-formvar coated grids and stained by inverting the grid on a droplet of $2 \%(\mathrm{w} / \mathrm{v}, \mathrm{pH} 4.5)$ aqueous solution of uranyl acetate to fix, wash off the salt and stain. Uranyl acetate preserved the tetrameric state of Sec A due to its ability to fix proteins in milliseconds [8] before any sodium formate could be removed. Specimens were observed on a Hitachi 7000 and Zeiss 10C transmission electron microscope operated at 100 and $80 \mathrm{kV}$, respectively. Selected micrographs were digitized using a Leafscan 45 microdensitometer at a $10 \mu \mathrm{m}$ scan step corresponding to 2.53 or $2.9 \AA /$ pixel at the specimen level and single particle analysis was carried out using the IMAGIC 5 software package [9]. Class averages from 4,500 single molecules were initially obtained by reference free multivariate statistical analysis classification followed by iterations of multi reference alignment and classification [10]. The final 3D reconstruction was surface rendered to a threshold corresponding to $400 \mathrm{kDa}$ assuming a protein density of $1.37 \AA^{3} / \mathrm{Da}$ using the VMD package [11]. 


\section{References}

[1] E. H. Manting, A. J. M. Driessen., Mol. Microbiol 37 (2000) 226

[2] A. J. M. Driessen., Trends Microbiol 9 (2001) 193

[3] J. F. Hunt, S et al., Science 297 (2002) 2018

[4] E. Or, A. Navon, T. Rapoport., EMBO 21 (2002) 4470

[5] B.R. Dempsey et al., J. Mol. Biol 315 (2002) 831

[6] C. Breyton et al., Nature 418 (2002) 662

[7] E.H. Manting et al., EMBO 19 (2000) 852

[8] F. Zhao, R. Craig., Microsc. Microanal. 8 (Suppl. 2) (2002) 828CD

[9] van Heel, M et al., J. Struct. Biol. 116 (1996) 17

[10] van Heel, M., Optik 82 (1989) 114

[11] W. Humphrey, A. Dalke, K. Schulten., J. Mol. Graph 14 (1996) 33

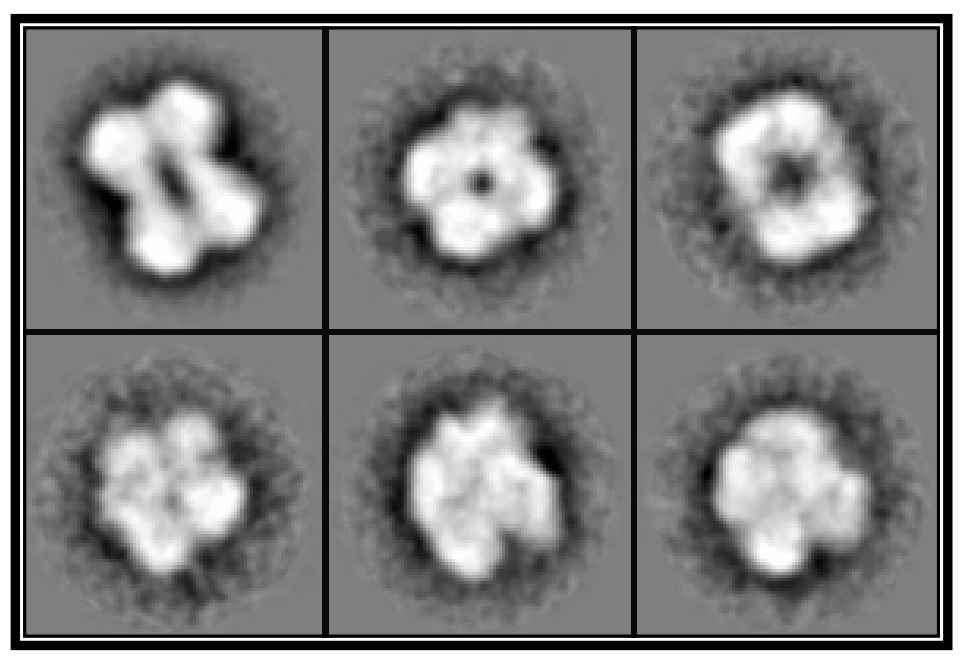

FIG 1. Class averages of $M t b$ SecA. Box size is $24 \mathrm{~nm}$.
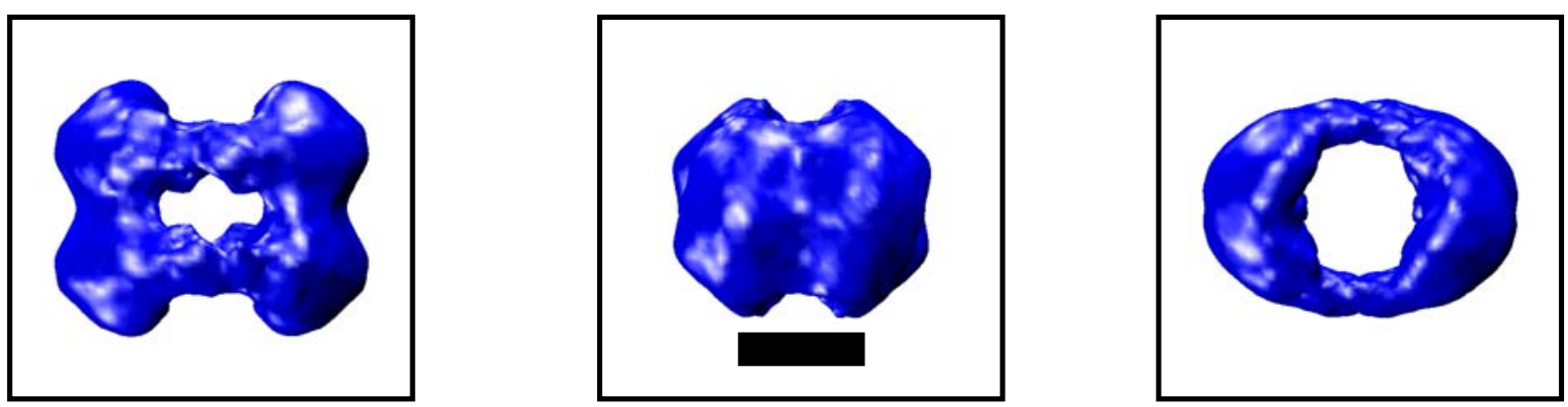

FIG 2. Three-dimensional reconstruction of $M t b$ SecA depicting the 3 orthogonal views which correspond to the top row class averages in fig 1 . Scale bar corresponds to $5 \mathrm{~nm}$. 